\title{
New onset seizures in children less than 2 years: is emergent CT imaging necessary?
}

\begin{abstract}
Background: Seizures being a common problem in paediatrics population especially in children less than 2 years old. Although Computed Tomography (CT) is invaluable in providing quick information for prompt clinical management, it has potential hazardous effects of an associated ionizing radiation which are concerning for frequent use. We are examining the yield of CT Brain findings in children less than 2 years old who presented with first episode of seizures without documented prior trauma. Materials and Methods: A cross sectional descriptive study based on retrospective data of children less than 2 years old who had undergone CT Brain examination in Hospital Serdang during first presentation of seizures from January 2012 till June 2015. The images were reviewed by 2 radiologists with special interest in Paediatric Radiology and any discrepancy was resolved by consensus. Comparative study between afebrile and febrile groups was made. Result: There were 377 CT Brain examinations performed in children less than 2 years old from January 2012 till June 2015, with 88 cases due to first episode of seizures without documented prior trauma. The age ranged between 1 to 23 months (mean 6.84 months, SD 5.223). A large proportion was represented by Malays $(\mathrm{N}=78$; $88.6 \%)$ followed by foreigners/migrants $(\mathrm{N}=5 ; 5.7 \%)$, Chinese $(\mathrm{N}=3 ; 3.4 \%)$ and Indian $(\mathrm{N}=2$; $2.3 \%$ ). Male preponderance was noted with 61 cases $(69.3 \%) .57$ of cases had febrile seizures, while 31 of cases had afebrile seizures. Using a chi-square test, afebrile seizures were found to have significant association with abnormal CT Brain findings ( $\mathrm{N}=25,80.6 \%$; $\mathrm{p}=0.005)$, intracranial bleed $(\mathrm{N}=16,48 \% ; \mathrm{p}=0.001)$ and suspected non-accidental injuries $(\mathrm{N}=11,35.5 \%$; $\mathrm{p}=0.001$ ). Conclusion: Afebrile seizures in children less than 2 years old without documented trauma signify ominous risk of having abnormal CT Brain findings, intracranial bleed and suspected non-accidental injuries thus necessitate emergent imaging at presentation.
\end{abstract}

Keyword: Seizures in children; CT scan 